\title{
Global Weak Solutions and Attractors of the Three Dimensional Maxwell-Bloch Two Level Laser Systems
}

\author{
J. Xin, J. Moloney \\ Department of Mathematics, Program in Applied Mathematics, University of Arizona, \\ Tucson, AZ 85721, USA
}

Received: 14 August 1995/Accepted: 3 December 1995

\begin{abstract}
The three-dimensional Maxwell-Bloch system governs the multi-longitudinal and transverse mode dynamics of two level wide aperture lasers in an optical ring cavity. The system is hyperbolic in the propagation direction, and dispersive in the transverse directions due to diffraction effects. A rich variety of optical patterns and chaos are present in the dynamics. We show the global existence of weak solutions in $L^{p}(2 \leqq p<\infty)$ spaces of the Maxwell-Bloch system under both absorbing and periodic boundary conditions. The weak solutions are unique within the class of solutions provided by our regularization procedure and approach a universal attractor which has only partial smoothing instead of the $C^{\infty}$ smoothing property found in early works for the (longitudinal) one-dimensional and (transverse) two-dimensional cases. The idea of the proof makes essential use of both the hyperbolicity and dispersivity of the system. In the case of periodic boundary condition, our result depends on a conjectural Strichartz inequality.
\end{abstract}

\section{Introduction}

In this paper, we are concerned with the dynamics of the three dimensional Maxwell-Bloch(MB) two level laser system:

$$
\begin{gathered}
\mathscr{E}_{t}+\mathscr{E}_{z}-i a \Delta_{\perp} \mathscr{E}=-\sigma \mathscr{E}+\sigma \mathscr{P}, \\
\mathscr{P}_{t}+(1+i \Omega) \mathscr{P}=(r-\mathscr{N}) \mathscr{E}, \\
\mathscr{N}_{t}+b \mathscr{N}=\frac{1}{2}\left(\mathscr{E}^{\star} \mathscr{P}+\mathscr{E}^{\star}\right),
\end{gathered}
$$

where $\Delta_{\perp}=$ the two dimensional Laplacian in $(x, y) \in R^{2}$ or $T^{2}$ ( $T^{n}$ will denote the unit $n$-dimensional torus $),(x, y)$ being the transverse dimensions; the real parameter $a$ measures the transverse diffraction; $z \in T^{1}$, the longitudinal or propagational dimension that has been normalized to size one. The complex variables $\mathscr{E}$ and $\mathscr{P}$ are the electric and polarization fields, and $\mathscr{N}$ is proportional to the difference between the atomic and the initial inversion; the positive parameters $\sigma$ and $b$ are respectively the dissipation(decay) rates of the electric field and population inversion both 
scaled to the decay rate of the polarization; the real detuning parameter $\Omega$ is the difference between the atomic and the cavity frequencies divided by the polarization decay rate; $r=r(x, y, z)$ is the external pumping function; and $\star$ denotes complex conjugate. For physical background and derivation of (1.1-1.3), we refer to Newell and Moloney [14].

The MB system reduces to the familiar complex Lorenz system if we ignore all the spatial dependence, so naturally $\mathrm{MB}$ is a chaotic system. This simple ode system is only realizable experimentally when imposed constraints such as curved mirrors, apertures, etc., prevent the development of spatiotemporal structures. This is achieved however at the expense of output power of the laser. Ideally one seeks spatially and temporally coherent high power laser output by removing many of these constraints and allowing the laser to be extended spatially in a direction transverse to the laser $(z)$ axis. Typically, there are two end mirrors located at $z=0$ and $z=1$. The laser turns on when enough energy is pumped externally, either incoherently (electrical discharge in a gas, broadband flashlamp for a solid rod, electrical voltage for a semiconductor, etc.) or, coherently (external laser beam). In the highly constrained geometry described by the complex Lorenz ode system (single longitudinal and transverse mode) the chaotic dynamics becomes nontrivial well above the first Hopf bifurcation (first laser threshold) and is difficult to realize experimentally. Transverse constraints can be removed by making the end reflectors flat and longitudinal constraints depend on the properties of the laser material itself. When removed a multitude of spectacular spatial temporal patterns due to the longitudinal $\left(\mathscr{E}_{z}\right)$ and the transverse $\left(\Delta_{\perp} \mathscr{E}\right)$ effects can emerge. Such lasers are of great technological significance in pattern recognition, space-based telecommunications, medical applications, etc., in circumstances where their spatiotemporal outputs can be exploited or controlled. For recent studies in the physics literature on pattern dynamics of lasers based on (1.1-1.3), we refer to [1,8,11-15], and references therein. The pumping function $r=r(x, y, z)$ normally has fast spatial decay in $(x, y)$, and its contribution is restricted to a bounded region, outside of which all the field variables can be regarded as zero. This motivates our absorbing boundary condition which we take to be $L_{x, y}^{2}\left(R^{2}\right) \times L^{2}\left(T^{1}\right)$ for convenience. The other is the commonly used periodic (in $(x, y, z)$ ) boundary condition $L^{2}\left(T^{3}\right)$. We will mainly consider these two boundary conditions in this paper, and comment on their physical modifications (see Lugiato et al. [13]) that can be handled with our method.

In the one-dimensional (in the longitudinal $z$ ) case, Constantin, Foias, and Gibbon [6] showed that the MB system admits a unique global weak $L^{2}$ solution for any $L^{2}$ periodic initial data; moreover, the weak solutions converge to a finite dimensional $C^{\infty}$ universal attractor as $t \rightarrow+\infty$. The MB system in this case becomes hyperbolic with two characteristic speeds, which is an essential ingredient of their work. In [6], the authors demonstrate the interesting time asymptotic $(t \rightarrow \infty)$ smoothing property of the MB system, even though the system is hyperbolic, and no second order dissipative effects are present. The dissipative terms are of zeroth order, and do not regularize dynamics. The smoothing instead comes from the special coupling of the polarization and the electric fields in MB.

Recently, Birnir and Xin [2] treated the two-dimensional transverse (in $x$ and $y$ ) case, and showed that the MB system admits unique global strong solutions in $H^{s}\left(T^{2}\right)(1<s \leqq 2)$ for periodic initial data; moreover, the strong solutions converge to a finite dimensional $C^{\infty}\left(T^{2}\right)$ attractor as $t \rightarrow \infty$. By strong we mean the solutions are imbedded into $L^{\infty}\left(T^{2}\right)$, and become classical $H^{2}\left(T^{2}\right)$ solutions if the initial data are in $H^{2}\left(T^{2}\right)$. Thanks to the coupling of $\mathscr{E}$ and $\mathscr{P}$, and the 
$L^{2}$ preserving group $\exp \left\{i t \Delta_{\perp}\right\}$, the two dimensional transverse case is even better than the one dimensional longitudinal case in that attracting sets exist for the MB dynamics in $H^{s}, 1<s \leqq 2$.

In the three-dimensional MB, we have an interaction of propagation in $z$ and diffraction in $(x, y)$, corresponding to an interplay of hyperbolic and dispersive aspects of the problem. Like in the case of the well-known three-D Navier-Stokes equations $([5,10,16])$, one is unable to show global existence of strong solutions to three-D MB with Sobolev imbedding. Instead, weak solutions are more appropriate. We first establish the existence of global smooth solutions to a regularized (MB) system, then show that the smooth solutions are strongly continuous with respect to the initial data for any finite time in the $L^{2}$ sense independent of the regularization. As in [6], this will allow us to construct the weak $L^{2}$ solutions to MB when we remove the regularization; moreover, the continuous dependence on the data yields the uniqueness of weak solutions within the class of solutions obtained by our regularization. We will see that a technical difficulty arises that prevents us from proving uniqueness among all weak solutions as in the one-D longitudinal or two-D transverse cases. The key step in our proof of continuous dependence is to estimate the nonlinear terms by Cauchy-Schwarz or Hölder inequalities and control different factors by combining the use of characteristic variable and the Strichartz inequality. The Strichartz inequality for the whole space $R^{2}$ is well-known, yet for a periodic domain $T^{2}$ it remains a conjecture, see Bourgain [3]. Our result in the periodic case will depend on Bourgain's conjecture. In both type of boundary conditions, the weak solutions and the attracting set are shown to stay in $(\mathscr{E}, \mathscr{P}, \mathscr{N}) \in L^{2} \times\left(L^{p} \cap L^{2}\right)^{2}$, for a suitable $p \in[4, \infty)$ coming from the Strichartz inequalities. The existence of a universal attractor follows for the unique global MB dynamics above; however, we find that it is only partially smoother than the initial data, i.e., it is smoother in terms of the directional derivative $\partial_{z}-i a \Delta_{\perp}$ but not the complete derivatives. This is very different from the one and two dimensional cases discussed in early works. In fact, the same partial smoothing occurs if we drop the $x$ or $y$ dependence, and the cause is the presence of both $\partial_{z}$ and $i a \Delta_{\perp}$ terms. During our proof, we recover the $C^{\infty}$ smoothing property of the attractor in the one-dimensional (in $z$ ) and two-dimensional transverse cases. As a byproduct, we extend the results in [2] from strong to weak solutions.

To be consistent with notations in early works [6 and 2], we make the change of variables $\mathscr{E}=X, \mathscr{P}=Y, \mathscr{N}=Z+r$ to rewrite $(1.1-1.3)$ as

$$
\begin{aligned}
X_{t}+X_{z}-i a \Delta_{\perp} X & =-\sigma X+\sigma Y, \\
Y_{t} & =-(1+i \Omega) Y-Z X, \\
Z_{t} & =-b Z+\operatorname{Re}\left(X Y^{\star}\right)-b r(x, y, z),
\end{aligned}
$$

where $r(x, y, z)$ is a smooth function with compact support. The initial condition for (1.4-1.6) is: $\left.(X, Y, Z)\right|_{t=0}=\left(X_{0}, Y_{0}, Z_{0}\right)$. Our main results are

Theorem 1.1. Let $\left(X_{0}, Y_{0}, Z_{0}\right)(x, y, z) \in\left(L^{2} \times\left(L^{2} \cap L^{4}\right)^{2}\right)\left(R^{2} \times T^{1}\right)$. Then there exists a global solution:

$$
S(t, \cdot)=(X(t, \cdot), Y(t, \cdot), Z(t, \cdot)), \quad \cdot=(x, y, z)
$$


of the system (1.4-1.6) such that

1) $S:[0,+\infty) \rightarrow L^{2} \times\left(L^{4} \cap L^{2}\right)^{2}\left(R^{2} \times T^{1}\right)$ is continuous and bounded;

2) $S$ satisfies the integral equations:

$$
\begin{aligned}
X(t, x, y, z) & =U(t) X_{0}(x, y, z-t)+\sigma \int_{0}^{t} U(t-s) Y(s, x, y, z-t+s) d s, \\
Y(t, \cdot) & =Y_{0}(\cdot)-(1+i \Omega) \int_{0}^{t} Y(s, \cdot) d s-\int_{0}^{t} Z(s, \cdot) X(s, \cdot) d s, \\
Z(t, \cdot) & =Z_{0}(\cdot)-b \int_{0}^{t} Z(s, \cdot) d s+\operatorname{Re} \int_{0}^{t} X Y^{\star}(s, \cdot) d s-r(\cdot) b t,
\end{aligned}
$$

where $U(t)=\exp \left\{-\right.$ iat $\left.\Delta_{\perp}-\sigma t\right\},(1.7)$ holds in $L^{2}\left(R^{2} \times T^{1}\right)$ and (1.8-1.9) holds in $\left(L_{\text {loc }}^{1}\left(R^{2} \times T^{1}\right)\right)^{2}$;

3) the solution $S(t, \cdot)$ is unique within the class of solutions obtained from our regularization (see Lemma 2.1) and the resulting unique $M B$ dynamics admits a universal attractor $\mathscr{A}$ in $\left(L^{2} \times\left(L^{2} \cap L^{4}\right)^{2}\right)\left(R^{2} \times T^{1}\right)$ such that if $(X, Y, Z) \in \mathscr{A}$ then $\left(\partial_{z}-i a \Delta_{\perp}\right) X \in L^{2}\left(R^{2} \times T^{1}\right)$ and $(Y, Z) \in\left(L^{\infty}\right)^{2}\left(R^{2} \times T^{1}\right)$;

4) if $S_{j}(0, \cdot) \stackrel{j \rightarrow \infty}{\longrightarrow} S(0, \cdot)$ in $L^{2}\left(R^{2} \times T^{1}\right)$, and $\left\|\left(Y_{j}(0, \cdot), Z_{j}(0, \cdot)\right)\right\|_{L^{4}} \leqq C$ as $j \rightarrow \infty$, for some finite constant $C<\infty$, then for any later time understood in the sense of 3 ):

in $\left(L^{2}\right)^{3}\left(R^{2} \times T^{1}\right)$ and

$$
S_{j}(t, \cdot) \stackrel{j \rightarrow \infty}{\longrightarrow} S(t, \cdot)
$$

$$
\|(Y(t, \cdot), Z(t, \cdot))\|_{L^{4}} \leqq C .
$$

We will present similar results for the periodic boundary conditions in the coming sections. Let us remark on the different features of the MB solutions for different dimensions. The two-D transverse (in $(x, y)$ ) case is the nicest in that it allows global classical solutions and attracting sets in $H^{2}$. The one-D longitudinal (in $z$ ) case also admits global classical $H^{1}$ solutions, however, the attracting set is not in $H^{1}$. Similarly the two-D longitudinal and transverse case (in $(x, z)$ or $\left.(y, z)\right)$ admits global classical solutions in $H^{2}$ but no attracting set even in $H^{1}$. The three-D MB is the worst case in that there seem to be no global classical solutions and there is only partial smoothing of the attractor. The three-D MB bears quite some resemblance to the three-D Navier-Stokes equations in terms of regularity properties of solutions (see [4]) and also in terms of how the usual method of proving classical solutions via the Sobolev imbedding fails. Fortunately, we have at least uniqueness of weak solutions within our regularization.

The rest of the paper is organized as follows. In Sect. 2, we give the proof of Theorem 1.1. In Sect. 3, we prove the analogue of Theorem 1.1 for the periodic boundary conditions. In Sect. 4, we analyze the partial smoothing property of the attractor $\mathscr{A}$, and comment on the inhomogeneous broadening effect and a nonperiodic boundary condition due to the imperfect reflection of end mirrors and finite cavity size.

\section{Three-Dimensional MB on Absorbing Domains}

In this section, we derive a-priori estimates for solution $(X, Y, Z)$ to the system (1.4-1.6), and use them to justify the existence and uniqueness of weak solutions 
as limits of smooth solutions to a regularized (MB) system. We will be brief with those estimates that follow directly from [6].

Multiplying (1.5) by $Y^{\star},(1.6)$ by $Z$, adding and taking the real part, we have

$$
\frac{1}{2}\left(|Y(t, \cdot)|^{2}+|Z(t, \cdot)|^{2}\right)_{t}=-|Y(t, \cdot)|^{2}-b|Z(t, \cdot)|^{2}-b r Z,
$$

which yields upon integrating in $t$ that

$$
|Y(t, \cdot)|^{2}+|Z(t, \cdot)|^{2} \leqq\left(\left|Y_{0}(\cdot)\right|^{2}+\left|Z_{0}(\cdot)\right|^{2}-b r^{2} / 2 \beta\right) e^{-2 \beta t}+b r^{2} / 2 \beta,
$$

where $\beta=\min \left(1, \frac{b}{2}\right)$, for any $t \geqq 0$, and any $(x, y, z)$. Integrating (2.2) over space gives

$$
\|Y\|_{2}^{2}+\|Z\|_{2}^{2} \leqq\left(\left\|Y_{0}\right\|_{2}^{2}+\left\|Z_{0}\right\|_{2}^{2}-b\|r\|_{2}^{2} / 2 \beta\right) e^{-2 \beta t}+b\|r\|_{2}^{2} / 2 \beta .
$$

It follows from (1.4) that

$$
X(t, \cdot)=e^{i a t \Delta_{\perp}-\sigma t} X_{0}(x, y, z-t)+\sigma \int_{0}^{t} e^{i a(t-s) \Delta_{\perp}-\sigma(t-s)} Y(s, x, y, z-t+s) d s .
$$

Taking the $L^{2}$ norm over space and using $e^{\text {iat } \Delta_{\perp}}$ being an $L^{2}$ isometry, we have

$$
\|X\|_{2} \leqq e^{-\sigma t}\left\|X_{0}\right\|_{2}+\sigma \int_{0}^{t} e^{-\sigma(t-s)}\|Y\|_{2}(s) d s,
$$

which gives as we plug in (2.3):

$$
\|X\|_{2}(t) \leqq e^{-\sigma t}\left\|X_{0}\right\|_{2}+\left(\frac{b\|r\|_{2}^{2}}{2 \beta}\right)^{\frac{1}{2}}+\sigma e(t)\left\|\left(Y_{0}, Z_{0}\right)\right\|_{2},
$$

where $e(t)=\left(e^{-\beta t}-e^{-\sigma t}\right) /(\beta-\sigma)$, if $\beta \neq \sigma$, and $e(t)=t e^{-\sigma t}$, if $\beta=\sigma$. Combining (2.3) and (2.6), we have the $L^{2}$ bounds:

$$
\|(X, Y, Z)\|_{2}(t) \leqq 2\left(\frac{b\|r\|_{2}^{2}}{2 \beta}\right)^{\frac{1}{2}}+\left\|\left(X_{0}, Y_{0}, Z_{0}\right)\right\|_{2} e^{-\min (\sigma, \beta) t}+\sigma e(t)\left\|\left(Y_{0}, Z_{0}\right)\right\|_{2} .
$$

Squaring (2.2) and integrating over space give the $L^{4}$ bounds:

$$
\|(Y, Z)\|_{4}(t) \leqq\left\|\left(Y_{0}, Z_{0}\right)\right\|_{4} e^{-\beta t}+C(b, \beta)\|r\|_{4} .
$$

Now as in [6], we proceed to estimate the difference of any two solutions $\left(X_{1}, Y_{1}, Z_{1}\right)$ and $\left(X_{2}, Y_{2}, Z_{2}\right)$ to system (1.4-1.6); and introduce their differences and averages:

$$
\begin{array}{r}
\xi=X_{1}-X_{2}, \quad \eta=Y_{1}-Y_{2}, \quad \zeta=Z_{1}-Z_{2}, \\
\tilde{X}=\frac{1}{2}\left(X_{1}+X_{2}\right), \quad \tilde{Y}=\frac{1}{2}\left(Y_{1}+Y_{2}\right), \quad \tilde{Z}=\frac{1}{2}\left(Z_{1}+Z_{2}\right) .
\end{array}
$$

The variables $(\xi, \eta, \zeta)$ solve the following linear system with coefficients $\tilde{X}, \tilde{Y}, \tilde{Z}$ :

$$
\begin{array}{r}
\xi_{t}+\xi_{z}-i a \Delta_{\perp} \xi=-\sigma \xi+\sigma \eta, \\
\eta_{t}=-(1+i \Omega) \eta-\tilde{X} \zeta-\tilde{Z} \xi, \\
\zeta_{t}=-b \zeta+\operatorname{Re}\left(\tilde{X} \eta^{\star}\right)+\operatorname{Re}\left(\xi \tilde{Y}^{\star}\right) .
\end{array}
$$


Inequality (2.2) implies that

$$
\left(|\tilde{Y}|^{2}+|\tilde{Z}|^{2}\right)(t, \cdot) \leqq h^{2}(\cdot), \quad \forall t \geqq 0,
$$

where

$$
h(\cdot)^{2}=b r^{2}(\cdot) / \beta+\sum_{i=1,2}\left(\left|Y_{0, i}(\cdot)\right|^{2}+\left|Z_{0, i}(\cdot)\right|^{2}\right) .
$$

We make the change of variables: $z^{\prime}=z-t,(t, x, y)=(t, x, y)$. Then $(2.11-2.13)$ become

$$
\begin{gathered}
\xi_{t}-i a \Delta_{\perp} \xi=-\sigma \xi+\sigma \eta, \\
\eta_{t}-\eta_{z^{\prime}}=-(1+i \Omega) \eta-\tilde{X} \zeta-\tilde{Z} \xi \\
\zeta_{t}-\zeta_{z^{\prime}}=-b \zeta+\operatorname{Re}\left(\tilde{X} \eta^{\star}\right)+\operatorname{Re}\left(\xi \tilde{Y}^{\star}\right) .
\end{gathered}
$$

In the new variables $\left(t, x, y, z^{\prime}\right),(2.14)$ reads:

$$
|\tilde{Y}|^{2}+|\tilde{Z}|^{2}\left(t, x, y, z^{\prime}\right) \leqq h^{2}\left(x, y, z^{\prime}+t\right)
$$

for any $t>0$, and $\left(x, y, z^{\prime}\right) \in R^{2} \times T^{1}$. It follows from $(2.15)$ that

$$
\xi\left(t, x, y, z^{\prime}\right)=e^{i a t \Delta_{\perp}-\sigma t} \xi_{0}\left(x, y, z^{\prime}\right)+\sigma \int_{0}^{t} e^{i a(t-s) \Delta_{\perp}-\sigma(t-s)} \eta\left(s, x, y, z^{\prime}\right) d s .
$$

Multiplying $(2.16)$ by $\eta^{\star},(2.17)$ by $\zeta$, adding and taking the real part shows:

$$
\frac{1}{2}\left(|\eta|^{2}+|\zeta|^{2}\right)_{t}-\frac{1}{2}\left(|\eta|^{2}+|\zeta|^{2}\right)_{z^{\prime}} \leqq-|\eta|^{2}-b|\zeta|^{2}+|\xi|(|\tilde{Z} \eta|+|\tilde{Y} \zeta|)
$$

or

$$
\begin{aligned}
\left(|\eta|^{2}+|\zeta|^{2}\right)_{t} \leqq & \left(|\eta|^{2}+|\zeta|^{2}\right)_{z^{\prime}}-|\eta|^{2}-b|\zeta|^{2} \\
& +\left(1+b^{-1}\right) h^{2}\left(x, y, z^{\prime}+t\right)|\xi|^{2}\left(t, x, y, z^{\prime}\right),
\end{aligned}
$$

which gives upon integrating in $t$ :

$$
\begin{aligned}
\left(|\eta|^{2}+|\zeta|^{2}\right)\left(t, x, y, z^{\prime}\right) \leqq & e^{-\beta t}\left(\left|\eta_{0}\right|^{2}+\left|\zeta_{0}\right|^{2}\right)+\int_{0}^{t} e^{-\beta(t-s)}\left(|\eta|^{2}+|\zeta|^{2}\right)_{z^{\prime}} d s \\
& +\left(1+b^{-1}\right) \int_{0}^{t} e^{-\beta(t-s)} h^{2}\left(x, y, z^{\prime}+s\right)|\xi|^{2}\left(s, x, y, z^{\prime}\right) d s
\end{aligned}
$$

The proper handling of the last integral is the key to achieving the estimates of $(\xi, \eta, \zeta)(t)$ in terms of their initial data. In [6], the authors exploit the two distinct characteristic speeds to separate $h^{2}$ and $|\xi|^{2}$ so as to bound the $L^{1}$ norm (in $z$ ) of the last integral in terms of the product of the $L^{2}$ norm square of $\xi$ and $h$. In fact, in their case, the original variables $(t, z)$ are better. However, in the presence of dispersion, we need to make use of both hyperbolicity and dispersivity, for which the new variables $\left(t, x, y, z^{\prime}\right)$ are more convenient. 
Integrating (2.22) over $(x, y) \in R^{2}$ to get with Cauchy-Schwarz inequality:

$$
\begin{aligned}
\|(\eta, \zeta)\|_{L^{2}\left(R^{2}\right)}^{2}\left(t, z^{\prime}\right) \leqq & e^{-\beta t}\left\|\left(\eta_{0}, \zeta_{0}\right)\right\|_{L^{2}\left(R^{2}\right)}^{2}+\int_{0}^{t} \int_{R^{2}} e^{-\beta(t-s)}\left(|\eta|^{2}+|\zeta|^{2}\right)_{z^{\prime}} \\
& +\left(1+b^{-1}\right)\left(\int_{0}^{t} \int_{R^{2}} e^{-\beta(t-s)} h^{4}\left(x, y, z^{\prime}+s\right)\right)^{1 / 2} \\
& \times\left(\int_{0}^{t} \int_{R^{2}} e^{-\beta(t-s)}|\xi|^{4}\left(t, x, y, z^{\prime}\right)\right)^{1 / 2}
\end{aligned}
$$

The first integral can be estimated by integrating $t$ over $[j, j+1], j=0,1, \ldots,[t]+1$, and summing up. Thanks to the hyperbolicity in the system, we pull out $\|h\|^{4}$ as below:

$$
\begin{aligned}
& \int_{0}^{t} \int_{R^{2}} e^{-\beta(t-s)} h^{4}\left(x, y, z^{\prime}+s\right)=\int_{R^{2}} \int_{0}^{t} e^{-\beta(t-s)} h^{4}\left(x, y, z^{\prime}+s\right) \\
& \quad \leqq \int_{R^{2}}\left(\sum_{j=0,[t]} \int_{j}^{j+1} h^{4}\left(x, y, z^{\prime}+s\right) e^{-\beta(t-s)} d s+\int_{[t]}^{[t]+1} h^{4}\left(x, y, z^{\prime}+s\right) d s\right) \\
& \quad=\int_{R^{2}}\left(\sum_{j=0,[t]} \int_{0}^{1} h^{4}\left(x, y, z^{\prime}+s\right) e^{-\beta(t-s-j)} d s+\int_{[t]}^{[t]+1} h^{4}(x, y, s) d s\right) \\
& \quad \leqq \int_{R^{2}}\left(\sum_{j=0,[t]} \int_{0}^{1} h^{4}(x, y, s) e^{-\beta(t-j)+\beta} d s+\int_{0}^{1} h^{4}(x, y, s) d s\right) \\
& \quad=\left(e^{\beta} \sum_{j=0,[t]} e^{-\beta(t-j)}+1\right)\|h\|_{L^{4}\left(R^{2} \times T^{1}\right)}^{4} \leqq C(\beta)\|h\|_{4}^{4}
\end{aligned}
$$

or

$$
\left(\int_{0}^{t} \int_{R^{2}} e^{-\beta(t-s)} h^{4}\left(x, y, z^{\prime}+s\right)\right)^{\frac{1}{2}} \leqq C(\beta)\|h\|_{4}^{2} .
$$

In view of (2.19), the second integral is bounded from above as:

$$
\begin{aligned}
\int_{0}^{t} d \tau \int_{R^{2}} e^{-\beta(t-s)}|\xi|^{4}\left(\tau, x, y, z^{\prime}\right) \leqq & C \int_{0}^{t} d \tau \int_{R^{2}}\left|e^{i a \tau \Delta_{\perp}-\sigma \tau} \xi_{0}\left(x, y, z^{\prime}\right)\right|^{4} \\
& +\sigma^{4} C \int_{0}^{t} d \tau \int_{R^{2}}\left|\int_{0}^{\tau} d s e^{i a(\tau-s) \Delta_{\perp}-\sigma(\tau-s)} \eta\left(s, x, y, z^{\prime}\right)\right|^{4}
\end{aligned}
$$


where $C$ is a universal constant. By changing variables in the PDE corresponding to the operator $e^{i a \tau \Delta_{\perp}-\sigma \tau}$, we infer from (2.26):

$$
\begin{aligned}
\int_{0}^{t} d \tau \int_{R^{2}} e^{-\beta(t-s)}|\xi|^{4}\left(\tau, x, y, z^{\prime}\right) \leqq & C \int_{0}^{t} d \tau \int_{R^{2}}\left|e^{i a \tau \Delta_{\perp}} \xi_{0}\left(x, y, z^{\prime}\right)\right|^{4} \\
& +\sigma^{4} C \int_{0}^{t} d \tau \int_{R^{2}}\left|\int_{0}^{\tau} d s e^{i a(\tau-s) \Delta_{\perp}} \eta\left(s, x, y, z^{\prime}\right) e^{\sigma s}\right|^{4} .
\end{aligned}
$$

Let us recall the Strichartz inequalities for the free Schrödinger operator (see Kato [9]):

$$
\left\|e^{i a t \Delta_{\perp}} \varphi(x, y)\right\|_{L^{p+1, r}} \leqq C\|\varphi\|_{L^{2}}, \quad \forall t \geqq 0,
$$

and

$$
\left\|\int_{0}^{t} e^{i a(t-s) \Delta_{\perp}} v(s, x, y) d s\right\|_{L^{p+1, r}} \leqq C \int_{0}^{t}\|v(s)\|_{L^{2}} d s, \quad \forall t \geqq 0,
$$

where $C$ is a universal constant; and $r=\frac{4(p+1)}{2(p-1)}, r \in(2,+\infty)$.

Let us choose $p=3, r=4$ in (2.28) and (2.29). So for any fixed $z^{\prime}$, we have

$$
\int_{0}^{t} d \tau \int_{R^{2}}\left|e^{i a \tau \Delta_{\perp}} \xi_{0}\left(x, y, z^{\prime}\right)\right|^{4} \leqq C\left\|\xi_{0}\left(x, y, z^{\prime}\right)\right\|_{L^{2}\left(R^{2}\right)}^{4},
$$

and

$$
\int_{0}^{t} d \tau \int_{R^{2}}\left|\int_{0}^{\tau} d s e^{i a(\tau-s) \Delta_{\perp}} \eta\left(s, x, y, z^{\prime}\right) e^{\sigma s}\right|^{4} \leqq C\left(\int_{0}^{t}\|\eta(s)\|_{L^{2}\left(R^{2}\right)} e^{\sigma s} d s\right)^{4} .
$$

Thus (2.27), (2.30), and (2.31) imply:

$$
\begin{aligned}
\int_{0}^{t} d \tau \int_{R^{2}} e^{-\beta(t-s)}|\xi|^{4}\left(\tau, x, y, z^{\prime}\right) \leqq & C\left\|\xi_{0}\left(x, y, z^{\prime}\right)\right\|_{L^{2}\left(R^{2}\right)}^{4}+C(\sigma) \\
& \times\left(\int_{0}^{t}\|\eta(s)\|_{L^{2}\left(R^{2}\right)} e^{\sigma s} d s\right)^{4},
\end{aligned}
$$

or

$$
\begin{aligned}
& \left(\int_{0}^{t} d \tau \int_{R^{2}} e^{-\beta(t-s)}|\xi|^{4}\left(\tau, x, y, z^{\prime}\right)\right)^{\frac{1}{2}} \\
& \leqq C\left\|\xi_{0}\left(x, y, z^{\prime}\right)\right\|_{L^{2}\left(R^{2}\right)}^{2}+C(\sigma)\left(\int_{0}^{t}\|\eta(s)\|_{L^{2}\left(R^{2}\right)} e^{\sigma s} d s\right)^{2} \\
& \leqq C\left\|\xi_{0}\left(x, y, z^{\prime}\right)\right\|_{L^{2}\left(R^{2}\right)}^{2}+C(\sigma) e^{2 \sigma t} t \int_{0}^{t}\|\eta(s)\|_{L^{2}\left(R^{2}\right)}^{2} d s
\end{aligned}
$$


Combining (2.23), (2.25) and (2.33), we get

$$
\begin{aligned}
\|(\eta, \zeta)\|_{L^{2}\left(R^{2}\right)}^{2}\left(t, z^{\prime}\right) \leqq & \left\|\left(\eta_{0}, \zeta_{0}\right)\right\|_{L^{2}\left(R^{2}\right)}^{2}+\int_{0}^{t} d s \int_{R^{2}} e^{-\beta(t-s)}\left(|\eta|^{2}+|\zeta|^{2}\right)_{z^{\prime}} \\
& +\left(1+b^{-1}\right) C(\beta)\|h\|_{L^{4}}^{2}\left(\left\|\xi_{0}\left(x, y, z^{\prime}\right)\right\|_{L^{2}\left(R^{2}\right)}^{2}\right. \\
& \left.+C(\sigma) t e^{2 \sigma t} \int_{0}^{t}\|\eta(s)\|_{L^{2}\left(R^{2}\right)}^{2} d s\right) .
\end{aligned}
$$

Integrating (2.34) over $z^{\prime} \in T^{1}$, we obtain

$$
\begin{aligned}
\|(\eta, \zeta)\|_{L^{2}}^{2}(t) \leqq & \left\|\left(\eta_{0}, \zeta_{0}\right)\right\|_{L^{2}}^{2}+\left(1+b^{-1}\right) C(\beta)\|h\|_{4}^{2}\left\|\xi_{0}\right\|_{2}^{2} \\
& +\left(1+b^{-1}\right) C(\sigma, \beta)\|h\|_{4}^{2} t e^{2 \sigma t} \int_{0}^{t}\|(\eta, \zeta)\|_{2}^{2} d s,
\end{aligned}
$$

which yields by Gronwall inequality:

$$
\begin{aligned}
\|(\eta, \zeta)\|_{L^{2}}^{2}(t) \leqq & \left(\left\|\left(\eta_{0}, \zeta_{0}\right)\right\|_{L^{2}}^{2}+\left(1+b^{-1}\right) C(\beta)\|h\|_{4}^{2}\left\|\xi_{0}\right\|_{2}^{2}\right) \\
& \times \exp \left\{t^{2} e^{2 \sigma t}\left(1+b^{-1}\right) C(\sigma, \beta)\|h\|_{4}^{2}\right\}
\end{aligned}
$$

for all $t \geqq 0$, where

$$
\|h\|_{4} \leqq C(b, \beta)\|r\|_{4}+C \sum_{i=1,2}\left\|\left(Y_{0, i}, Z_{0, i}\right)\right\|_{4} .
$$

Clearly, estimate (2.36) is also valid in the original variables $(t, x, y, z)$. Plugging (2.36) into (2.19) gives the desired bound on $\|\xi\|_{2}^{2}$ in terms of the $L^{2}$ norm of the initial data $\left(\xi_{0}, \eta_{0}, \zeta_{0}\right)$. Let us now study solutions to a regularized (MB) system in

Lemma 2.1. Consider the following regularized MB system:

$$
\begin{array}{r}
X_{t}+X_{z}-i a \Delta_{\perp} X=-\sigma X+\sigma Y \star \varphi_{\varepsilon}, \\
Y_{t}=-(1+i \Omega) Y-Z X, \\
Z_{t}=-b Z+\operatorname{Re}\left(X Y^{\star}\right)-b r(x, y, z),
\end{array}
$$

with $\varphi_{\varepsilon}$ the usual smooth mollifier converging to the delta-function as $\varepsilon \rightarrow 0$. Then for any initial data $\left(X_{0}^{\varepsilon}, Y_{0}^{\varepsilon}, Z_{0}^{\varepsilon}\right)=\left(X_{0} \star \varphi_{\varepsilon}, Y_{0} \star \varphi_{\varepsilon}, Z_{0} \star \varphi_{\varepsilon}\right)$ with $\left(X_{0}, Y_{0}, Z_{0}\right) \in\left(L^{2}\right)^{3}$, there exist unique solutions $\left(X^{\varepsilon}, Y^{\varepsilon}, Z^{\varepsilon}\right) \in C\left(R ; H^{k}\right)$, for any $k \geqq 2$, to $(2.38-2.40)$. Moreover, estimates (2.2), (2.7), (2.8), and (2.36) hold independent of $\varepsilon \in(0,1]$.

Proof. Let us write down the integral equations:

$$
\begin{aligned}
& X(t, \cdot)=\hat{U}(t) X_{0}^{\varepsilon}+\sigma \int_{0}^{t} \hat{U}(t-s)\left(Y \star \varphi_{\varepsilon}\right) d s \\
& Y(t, \cdot)=Y_{0}^{\varepsilon}-(1+i \Omega) \int_{0}^{t} Y(s, \cdot) d s-\int_{0}^{t} Z(s, \cdot) X(s, \cdot) d s \\
& Z(t, \cdot)=Z_{0}^{\varepsilon}-b \int_{0}^{t} Z(s, \cdot) d s+\operatorname{Re} \int_{0}^{t} X(s, \cdot) Y^{\star}(s, \cdot) d s-b r(\cdot) t
\end{aligned}
$$


where $\hat{U}(t) X_{0}^{\varepsilon}=e^{i a t \Delta_{\perp}-\sigma t} X_{0}^{\varepsilon}(x, y, z-t)$, and $\cdot=(x, y, z)$. It is straightforward to verify that the operator $T$ defined by the right-hand side of system (2.41) is a contraction mapping in $C\left(\left[0, t^{\star}\right) ;\left(H^{k}\right)^{3}\right), k \geqq 2$, if $t^{\star}=t^{\star}\left(\left\|\left(X_{0}^{\varepsilon}, Y_{0}^{\varepsilon}, Z_{0}^{\varepsilon}\right)\right\|_{k}\right)$ is small enough. Hence we have local in time solutions to (2.41). Such solutions also satisfy the differential system $(2.38-2.40)$ in the $L^{2}$ sense, and $(2.39-2.40)$ holds a.e. in $(x, y, z)$ for all $t \geqq 0$. Our a-priori estimates (2.2), (2.7), (2.8), and (2.36) apply to $\left(X^{\varepsilon}, Y^{\varepsilon}, Z^{\varepsilon}\right)$ independent of $\varepsilon \in(0,1]$. Based on these $L^{2}$ bounds, we differentiate (2.41) in $(x, y, z)$ and derive bounds on $\left\|\partial^{\alpha}(X, Y, Z)\right\|_{2},|\alpha|=1,2, \ldots, k$, inductively by the Gronwall inequality. Thanks to the regularization in (2.38), any spatial derivative of $X$ can be bounded by $\|Y\|_{2}$ with an $\varepsilon$ dependent bound. This and Sobolev imbedding allow us to overcome the nonlinearity $Z X$ and $X Y^{\star}$. We obtain then global strong solutions to $(2.38-2.40)$. The proof is complete.

We are ready for:

Proof of Theorem 1.1. By Lemma 2.1, and in particular the estimate (2.36), we see that for each $t,\left(X^{\varepsilon}, Y^{\varepsilon}, Z^{\varepsilon}\right)$ is a Cauchy sequence in $\left(L^{2}\right)^{3}\left(R^{2} \times T^{1}\right)$. As $\varepsilon \rightarrow$ 0 , the strong $\left(L^{2}\right)^{3}$ limit of $\left(\left(X^{\varepsilon}, Y^{\varepsilon}, Z^{\varepsilon}\right)\right.$ denoted by $(X, Y, Z)$ satisfies the integral equations (1.7-1.9) in $L^{2} \times\left(L_{\text {loc }}^{1}\right)^{2}\left(R^{2} \times T^{1}\right)$. Strong continuity of $S(t)$ follows from weak continuity and continuity of the norm. The weak solutions obtained from the regularization satisfy inequality (2.36), hence depend continuously on the data and are unique within our regularization. By adapting the a-priori estimates above, we easily see that the weak solutions are independent of the mollifier function $\varphi$ and the way $\varepsilon$ tends to zero. Moreover, estimates (2.7-2.8) imply an attracting ball for the MB dynamics in $L^{2} \times\left(L^{2} \cap L^{4}\right)^{2}$. We complete the proof except for the attractor and its partial smoothness which we defer to Sect. 4 .

Remark 2.1. We may follow the a-priori estimates (2.1-2.36) to study uniqueness of any weak solution. We infer from the second and third integral equations for $(Y, Z)$ components of weak solutions that (2.1) holds a.e. in $(x, y, z)$ for all $t$ which then leads to (2.2) and (2.14) (a.e. in $(x, y, z)$ ). Notice that so far, we do not need the equation for $\xi$. However, (2.22) should be in a different form:

$$
\int_{0}^{t}\left(\partial_{t}-\partial_{z}\right)\left(|\eta|^{2}+|\zeta|^{2}\right) \leqq\left(1+b^{-1}\right) \int_{0}^{t} h^{2}\left(x, y, z^{\prime}+s\right)|\xi|^{2}\left(s, x, y, z^{\prime}\right) d s
$$

where $\partial_{t}-\partial_{z}$ is understood as a directional derivative and not a difference of two partial derivatives, since we do not know they exist separately. In deriving (2.42), we also drop the term $-|\eta|^{2}-b|\zeta|^{2}$, as it turns out that omitting this term will not change the uniqueness proof, although it does affect the bounds. Now proceeding as before with the right-hand side in (2.42) using only the integral form of the $\xi$ equation, we have

$$
\int_{0}^{t} \int_{x, y, z^{\prime}}\left(\partial_{t}-\partial_{z^{\prime}}\right)\left(|\eta|^{2}+|\zeta|^{2}\right) \leqq C(t, b, \sigma, \beta) \int_{0}^{t}\|(\eta, \zeta)\|_{2}^{2} d s .
$$

The left-hand side of (2.43) is just

$$
\int_{0}^{t} \int_{x, y, z} \frac{d}{d t}\left(|\eta|^{2}+|\zeta|^{2}\right)
$$


by change of variable to $(t, x, y, z)$. However, we do not know if we can interchange the integration over $(x, y, z)$ and $\frac{d}{d t}$ since the time derivative of $|\eta|^{2}+|\zeta|^{2}$ exists only a.e. in $(x, y, z)$. If this could be done for all $t$, then it would not be hard to show that the time derivative of $\|(\eta, \zeta)\|_{2}^{2}$ is in $L^{1}$ and we would integrate out $\|(\eta, \zeta)\|_{2}^{2}$ and prove uniqueness by the Gronwall inequality. In the transverse two-D case, $z$ dependence drops out, and we get out $|\eta|^{2}+|\zeta|^{2}$ by integrating (2.21) in $t$ for a.e. $(x, y)$, since $|\eta|^{2}+|\zeta|^{2}$ is Lipschitz hence absolutely continuous in $t$ for a.e. $(x, y)$. So (2.22) is valid without the first integral on the right-hand side. Uniqueness then follows from the Gronwall inequality.

\section{Three-Dimensional MB on Periodic Domains}

In this section, we extend our results in Sect. 2 to the MB system (1.4-1.6) on $T^{3}$, including the special case when either $x$ or $y$ is absent (which is the $2 \mathrm{D} \mathrm{MB}$ with both longitudinal propagation and transverse diffraction). All the estimates up to (2.27) remain the same; however, the Strichartz inequalities are no longer valid for periodic domains when $p=3, r=4$. Even if they are valid for other powers of $p, r$, they hold only locally in time. We refer to the recent work of J. Bourgain [3] for a detailed account of the modified Strichartz inequalities. Some of the Bourgain estimates related to our analysis are:

1) If $f(x)=\sum_{n \in \mathscr{Z}} a_{n} e^{i n x} \in L^{2}\left(T^{1}\right)$, then

$$
\left\|\sum_{n \in \mathscr{Z}} a_{n} e^{i\left(n x+n^{2} t\right)}\right\|_{L^{4}\left(T^{2}\right)} \leqq 2\left(\sum_{n \in \mathscr{Z}}\left|a_{n}\right|^{2}\right)^{\frac{1}{2}},
$$

or

$$
\left\|e^{i t \partial_{x x}} f(x)\right\|_{L^{4}\left(T^{2}\right)} \leqq 2\|f\|_{2}
$$

Similar estimates are conjectured to be true with $L^{4}$ replaced by $L^{p}, p<6$; while it is known ([3]) if $p \geqq 6$, such inequalities break down.

2) If $f(x, y) \in L^{2}\left(T^{2}\right)$, then it is conjectured that

$$
\left\|e^{i t \Delta_{x, y}} f(x, y)\right\|_{L^{p}\left(T^{3}\right)} \leqq c_{p}\|f\|_{2}, \quad \text { if } p<4 \text {. }
$$

Estimates like (3.2) break down if $p \geqq 4$.

To apply (3.1-3.2), we write them as in [3]:

$$
\left\|e^{i t \Delta_{\perp}} f\right\|_{L^{p}\left(T^{d}\right)} \leqq c_{p}\|f\|_{2}, \quad p<\frac{2(d+1)}{d-1}
$$

where $d$ is the space plus time dimension; $d=2$ if $x \in T^{1}$, and $d=3$ if $x \in T^{2}$. We keep in mind that $p=4, d=2$ is proven and $p<4, d=3$ is still a conjecture.

Let us convert (3.3) into a convenient form for our application. First (3.3) reads:

$$
\int_{0}^{2 \pi} d t \int_{T^{d-1}} d x\left|e^{i t \Delta_{\perp}} f\right|^{p} \leqq c_{p}^{p}\|f\|_{2}^{p},
$$


so for $T>2 \pi$ and $n=[T / 2 \pi]+1$ :

$$
\begin{aligned}
\int_{0}^{T} d t \int_{T^{d-1}} d x\left|e^{i t \Delta_{\perp}} f\right|^{p} & \leqq \int_{0}^{2 \pi n} d t \int_{T^{d-1}} d x\left|e^{i t \Delta_{\perp}} f\right|^{p} \\
& \leqq \sum_{j=0, n} \int_{2 \pi(j-1)}^{2 \pi j} d t \int_{T^{d-1}} d x\left|e^{i(t-2 \pi(j-1)) \Delta_{\perp}} e^{i 2 \pi(j-1) \Delta_{\perp}} f\right|^{p} \\
& =\sum_{j=0, n} \int_{0}^{2 \pi} d t \int_{T^{d-1}} d x\left|e^{i t \Delta_{\perp}} e^{i 2 \pi(j-1) \Delta_{\perp}} f\right|^{p} \\
& \leqq \sum_{0}^{n} c_{p}^{p}\left\|e^{i 2 \pi(j-1) \Delta_{\perp}} f\right\|_{2}^{p} \quad(\text { applying }(3.3)) \\
& =\sum_{0}^{n} c_{p}^{p}\|f\|_{2}^{p}=\frac{n(n+1)}{2} c_{p}^{p}\|f\|_{2}^{p} .
\end{aligned}
$$

It follows that for any $T>0$ :

$$
\int_{0}^{T} d t \int_{T^{d-1}} d x\left|e^{i t \Delta_{\perp}} f\right|^{p} \leqq \frac{(T+4 \pi)^{2}}{8 \pi^{2}} c_{p}^{p}\|f\|_{2}^{p} .
$$

Then (3.5) and the Minkowski inequality imply the analogue of (2.29) as below:

$$
\begin{aligned}
& \left\|\int_{0}^{t} e^{i(t-s) \Delta_{\perp}} v(s, x)\right\|_{L^{p}\left(T^{d-1} \times[0, t]\right)}^{p}=\int_{0}^{t} d \tau \int_{T^{d-1}} d x\left|\int_{0}^{\tau} e^{i(\tau-s) \Delta_{\perp}} v(s, x) d s\right|^{p} \\
& \quad=\int_{0}^{t} d \tau\left[\left(\int_{T^{d-1}} d x\left|\int_{0}^{\tau} e^{i(\tau-s) \Delta_{\perp}} v(s, x) d s\right|^{p}\right)^{\frac{1}{p}}\right]^{p} \\
& \leqq \int_{0}^{t} d \tau\left[\int_{0}^{\tau} d s\left(\int_{T^{d-1}} d x\left|e^{i(\tau-s) \Delta_{\perp}} v(s, x)\right|^{p}\right)^{\frac{1}{p}}\right]^{p} \\
& \left.\quad=\left(\int_{0}^{t} d \tau\left[\int_{0}^{\tau} d s\left(\int_{T^{d-1}} d x\left|e^{i(\tau-s) \Delta_{\perp}} v(s, x)\right|^{p}\right)^{\frac{1}{p}}\right]^{p}\right\}^{\frac{1}{p}}\right)^{p} \\
& \leqq\left(\int_{0}^{t} d s\left[\int_{s}^{t} d \tau \int_{T^{d-1}} d x\left|e^{i(\tau-s) \Delta_{\perp}} v(s, x)\right|^{p}\right]^{\frac{1}{p}}\right)^{p} \\
& \leqq \\
& \quad \frac{(t+4 \pi)^{2}}{8 \pi^{2}} c_{p}^{p}\left(\int_{0}^{t} d s\|v(s)\|_{2}\right)^{p},
\end{aligned}
$$

which implies

$$
\left\|\int_{0}^{t} e^{i(t-s) \Delta_{\perp}} v(s, x)\right\|_{L^{p}\left(T^{d-1} \times[0, t]\right)} \leqq \frac{(t+4 \pi)^{2}}{8 \pi^{2}} c_{p} \int_{0}^{t} d s\|v(s)\|_{2}, \quad \forall t \geqq 0 .
$$

In case of the two-dimensional MB in $(x, z)$ or $(y, z), \Delta_{\perp}=\frac{\partial^{2}}{\partial x^{2}}$ or $\frac{\partial^{2}}{\partial y^{2}}$, we are in the $d=2$ regime, and so we take $p=4$. Employing (3.5) and (3.7), we obtain as before (2.36), except that the coefficients $C(\beta)$ and $C(\sigma)$ now depend on 
time $t$ continuously due to the time dependent bounds in (3.5) and (3.7). All the rest remains the same and we arrive at a similar global existence and uniqueness theorem for weak solutions with the space $L^{2} \times\left(L^{2} \cap L^{4}\right)^{2}\left(R^{2} \times T^{1}\right)$ replaced by $L^{2} \times\left(L^{4}\right)^{2}\left(T^{2}\right)$, and $L_{\text {loc }}^{1}$ by $L^{1}$ in Theorem 1.1 .

In case of the three-dimensional $\mathrm{MB}$ on $T^{3}$, let us assume that (3.3) holds for some $p^{\star} \in(2,4)$. Then we go back to $(2.22)$ and use the Hölder inequality to derive:

$$
\begin{aligned}
\|(\eta, \zeta)\|_{L^{2}\left(T^{2}\right)}^{2}\left(t, z^{\prime}\right) \leqq & e^{-\beta t}\left\|\left(\eta_{0}, \zeta_{0}\right)\right\|_{L^{2}\left(T^{2}\right)}^{2}+\int_{0}^{t} \int_{T^{2}} e^{-\beta(t-s)}\left(|\eta|^{2}+|\zeta|^{2}\right)_{z^{\prime}} \\
& +\left(1+b^{-1}\right)\left(\int_{0}^{t} \int_{T^{2}} e^{-\beta(t-s)} \frac{2 p^{\star}}{p^{\star}-2}\left(x, y, z^{\prime}+s\right)\right)^{\frac{p^{\star}-2}{p^{\star}}} \\
& \times\left(\int_{0}^{t} \int_{T^{2}} e^{-\beta(t-s)}|\xi|^{p^{\star}}\left(t, x, y, z^{\prime}\right)\right)^{\frac{2}{p^{\star}}}
\end{aligned}
$$

The first integral is bounded as before:

$$
\left(\int_{0}^{t} \int_{T^{2}} e^{-\beta(t-s)} h^{\frac{2 p^{\star}}{p^{\star}-2}}\left(x, y, z^{\prime}+s\right)\right)^{\frac{p^{\star}-2}{p^{\star}}} \leqq C\left(\beta, p^{\star}\right)\|h\|_{\frac{2 p^{\star}}{p^{\star}-2}}^{2} .
$$

The second integral is bounded with (3.5) and (3.7) as

$$
\int_{0}^{t} \int_{T^{2}} e^{-\beta(t-s)}|\xi|^{p^{\star}}\left(t, x, y, z^{\prime}\right) \leqq C_{1}(t)\left\|\xi_{0}\left(x, y, z^{\prime}\right)\right\|_{L^{2}\left(T^{2}\right)}^{p^{\star}}+C_{2}(t)\left(\int_{0}^{t}\|\eta(s)\|_{L^{2}\left(T^{2}\right)} d s\right)^{p^{\star}}
$$

which yields

$$
\begin{aligned}
\left(\int_{0}^{t} \int_{T^{2}} e^{-\beta(t-s)}|\xi|^{p^{\star}}\left(t, x, y, z^{\prime}\right)\right)^{\frac{2}{p^{\star}}} \leqq & C_{1}(t)\left\|\xi_{0}\left(x, y, z^{\prime}\right)\right\|_{L^{2}\left(T^{2}\right)}^{2} \\
& +C_{2}(t) \int_{0}^{t}\|\eta(s)\|_{L^{2}\left(T^{2}\right)}^{2} d s .
\end{aligned}
$$

Substituting (3.9) and (3.11) into (3.8) and integrating in $z^{\prime}$ gives

$$
\begin{aligned}
\|(\eta, \zeta)\|_{L^{2}\left(T^{3}\right)}^{2}(t) \leqq & \left\|\left(\eta_{0}, \zeta_{0}\right)\right\|_{L^{2}\left(T^{3}\right)}^{2}+C_{1}(t)\|h\|_{\frac{2 p^{\star}}{p^{\star}-2}}^{2}\left\|\zeta_{0}\right\|_{L^{2}\left(T^{3}\right)}^{2} \\
& +C_{2}(t)\|h\|_{\frac{2 p^{\star}}{p^{\star}-2}}^{2} \int_{0}^{t}\|\eta(s)\|_{L^{2}\left(T^{3}\right)}^{2} d s,
\end{aligned}
$$

from which we conclude with Gronwall inequality. Summarizing, we have:

Theorem 3.1. Let $\left(X_{0}, Y_{0}, Z_{0}\right)(x, y, z) \in L^{2} \times\left(L^{q^{\star}}\right)^{2}\left(T^{3}\right), \quad q^{\star}=\frac{2 p^{\star}}{p^{\star}-2}, \quad$ where $p^{\star} \in(2,4)$ is the exponent for which the conjectural Strichartz inequality (3.3) is valid. Then there exists a global solution:

$$
S(t, \cdot)=(X(t, \cdot), Y(t, \cdot), Z(t, \cdot)), \cdot=(x, y, z)
$$


of the system (1.4-1.6) such that:

1) $S:[0,+\infty) \rightarrow L^{2} \times\left(L^{q^{\star}}\right)^{2}\left(T^{3}\right)$ is continuous and bounded.

2) $S$ satisfies the integral equations (1.7-1.9), (1.7) holds in $L^{2}\left(T^{3}\right)$ and (1.81.9) hold in $\left(L^{1}\left(T^{3}\right)\right)^{2}$.

3) $S$ is unique within the class of solutions constructed by our regularization and estimate (2.2) holds a.e. in $(x, y, z)$, any $t \geqq 0$; so does (2.7) for $t \geqq 0$; in particular, there exists an absorbing ball of the $\mathrm{MB}$ dynamics in $L^{2} \times\left(L^{q^{\star}}\right)^{2}\left(T^{3}\right)$.

4) If $S_{j}(0, \cdot) \stackrel{j \rightarrow \infty}{\longrightarrow} S(0, \cdot)$ in $L^{2}\left(T^{3}\right)$, and $\left\|\left(Y_{j}(0, \cdot), Z_{j}(0, \cdot)\right)\right\|_{L^{q^{\star}}\left(T^{3}\right)} \leqq C$ as $j \rightarrow \infty$, for finite constant $C<\infty$, then for any later time understood in the sense of 3$)$ :

$$
S_{j}(t, \cdot) \stackrel{j \rightarrow \infty}{\longrightarrow} S(t, \cdot)
$$

in $\left(L^{2}\right)^{3}\left(T^{3}\right)$ and

$$
\|(Y(t, \cdot), Z(t, \cdot))\|_{L^{q^{\star}}\left(T^{3}\right)} \leqq C .
$$

5) If $\left(X_{0}, Y_{0}, Z_{0}\right)$ is independent of $x$ or $y$, then the above conclusions 1)-4) are true with $q^{\star}=4$ on $T^{2}$ without the conjectural assumption on $p^{\star}(=4)$.

\section{Partial Smoothing of the Universal Attractor}

In this section, we discuss the regularity of universal attractor $\mathscr{A}$ of the MB system. Theorems 1.1 and 3.1 show that $S(t)$ is a group of continuous maps in $L^{2} \times\left(L^{p} \cap\right.$ $\left.L^{2}\right)^{2}$, with $p=4$ or $p=q^{\star}>4$. This and the existence of the absorbing set

$$
B_{\rho_{0}}=\left\{(X, Y, Z):\|(X, Y, Z)\|_{L^{2} \times\left(L^{p} \cap L^{2}\right)^{2}} \leqq \rho_{0}\right\},
$$

where $\rho_{0}$ depends only on the coefficients of $\mathrm{MB}$, ensures the existence of the universal attractor $\mathscr{A}$ defined as the intersection of all $S(t) B_{\rho_{0}}, t>0$. The $\mathscr{A}$ is the largest invariant set of the MB dynamics in $L^{2} \times\left(L^{p} \cap L^{2}\right)^{2}$, and all trajectories $S(t)\left(X_{0}, Y_{0}, Z_{0}\right)$ eventually approach $\mathscr{A}$. We refer to [6] for the basic properties of the universal attractor $\mathscr{A}$.

We will treat the periodic case below. Easy adaptation with Fourier transform gives the same result for the absorbing domain $R^{2} \times T^{1}$. As a byproduct, we will show that $\mathscr{A}$ is $C^{\infty}$ for the two-dimensional transverse (in $(x, y)$ ) MB system on $T^{2}$, thereby extending the result in [2] to smoothing of $\mathscr{A}$ on rough data. We state

Theorem 4.1. The universal attractor $\mathscr{A}$ for the three dimensional $\mathrm{MB}$ dynamics on $T^{3}$ or $R^{2} \times T^{1}$ is included in a subspace of $L^{2} \times\left(L^{p} \cap L^{2}\right)^{2}$ defined as

$$
\mathscr{P}=\left\{(X, Y, Z) \in L^{2} \times\left(L^{p} \cap L^{2}\right)^{2} \mid-i a \Delta_{\perp} X+X_{z} \in L^{2},(Y, Z) \in\left(L^{\infty}\right)^{2}\right\} .
$$

Proof. We first show that for each $V \equiv(X, Y, Z) \in \mathscr{A}$, the last two components $(Y, Z) \in\left(L^{\infty}\left(T^{3}\right)\right)^{2}$. This part is the same as in [6] except that we can not say anything about the maximum norm of $X$ due to the presence of the dispersion term $i a \Delta_{\perp}$. Since $V \in \mathscr{A}$, then for any $t>0$, there exists $V_{0}=\left(X_{0}, Y_{0}, Z_{0}\right) \in B_{\rho_{0}}\left(V_{0}\right.$ is time dependent) such that $V=S(t) V_{0}$. We have from (2.2):

$$
|(Y, Z)(x, y, z)|^{2} \leqq M^{2}+e^{-2 \beta t}\left(\left|Y_{0}\right|^{2}+\left|Z_{0}\right|^{2}\right)(x, y, z),
$$


where $M$ depends only on the coefficients of MB. Let

$$
\Sigma=\left\{(x, y, z) \in T^{3}:|(Y, Z)|(x, y, z) \geqq 2 M\right\},
$$

then on $\Sigma$

$$
3 M^{2} \leqq e^{-2 \beta t}\left(\left|Y_{0}\right|^{2}+\left|Z_{0}\right|^{2}\right)(x, y, z),
$$

which upon intergating over $\Sigma$ gives

$$
3|\Sigma| M^{2} \leqq e^{-2 \beta t}||\left(Y_{0}, Z_{0}\right) \|_{2}^{2} \leqq e^{-2 \beta t} \rho_{0}^{2} .
$$

Sending $t \rightarrow+\infty$ yields $|\Sigma|=0$, and $(Y, Z) \in\left(L^{\infty}\right)^{2}$.

Next we show that if $V=(X, Y, Z) \in \mathscr{A}$, then its first component $X$ satisfies $-i a \Delta_{\perp} X+X_{z} \in L^{2}\left(T^{3}\right)$. Write $V=S(t) V_{0}$ for some $V_{0} \in B_{\rho_{0}} ;$ so $V(s)=S(s) V_{0}=$ $S(s-t) V, \forall s \in[0, t]$, lies on the attractor $\mathscr{A}$. By (2.4):

$$
\begin{aligned}
X(x, y, z)= & e^{i a t \Delta_{\perp}-\sigma t} X_{0}(x, y, z-t) \\
& +\sigma \int_{0}^{t} e^{i a(t-s) \Delta_{\perp}-\sigma(t-s)} Y(s, x, y, z-t+s) d s,
\end{aligned}
$$

where $Y(s, \cdot)$ is the second component of $S(s) V_{0}=S(s-t) V$, which implies that

$$
Y(s, \cdot)=V_{0}-(1+i \Omega) \int_{0}^{s} Y(\tau, \cdot) d \tau-\int_{0}^{s} Z(\tau, \cdot) X(\tau, \cdot) d \tau,
$$

where $Z(\tau, \cdot)$ is in $L^{\infty}\left(T^{3}\right)$ by what we just showed above. Hence,

$$
\left\|Y_{s}(s, \cdot)\right\|_{2} \leqq\left(1+\Omega^{2}\right)^{\frac{1}{2}} \rho_{0}+\|Z\|_{\infty} \rho_{0} \equiv M_{1}<+\infty, \forall s \geqq 0,
$$

where $M_{1}$ depends only on the attractor $\mathscr{A}$. Now we write $Y(s, \cdot), Y_{s}(s, \cdot)$ into a Fourier series:

$$
\begin{aligned}
& Y(s, x, y, z)=\sum_{k} y_{k}(s) e^{\imath k \cdot(x, y, z)}, \\
& Y_{s}(s, x, y, z)=\sum_{k} y_{k}^{\prime}(s) e^{i k \cdot(x, y, z)},
\end{aligned}
$$

where $k=\left(k_{1}, k_{2}, k_{3}\right) \in(2 \pi \mathscr{Z})^{3}$, and prime denotes $\frac{d}{d s}$. We also denote $k_{\perp}=$ $\left(k_{1}, k_{2}\right)$. It follows that

$$
\begin{aligned}
I \equiv & \int_{0}^{t} e^{i a(t-s) \Delta_{\perp}-\sigma(t-s)} Y(s, x, y, z-t+s) d s \\
= & \sum_{k} \int_{0}^{t} e^{-i a(t-s)\left|k_{\perp}\right|^{2}-\sigma(t-s)} y_{k}(s) e^{i k \cdot(x, y, z)+i k_{3}(-t+s)} d s \\
= & \sum_{k} e^{i k \cdot(x, y, z)} \int_{0}^{t} y_{k}(s) d \frac{e^{-i a(t-s)\left|k_{\perp}\right|^{2}-\sigma(t-s)+i k_{3}(-t+s)}}{i a\left|k_{\perp}\right|^{2}+\sigma+i k_{3}} \\
= & \sum_{k} e^{i k \cdot(x, y, z)}\left[\frac{y_{k}(t)-y_{k}(0) e^{-i a t\left|k_{\perp}\right|^{2}-\sigma t-i k_{3} t}}{i a\left|k_{\perp}\right|^{2}+\sigma+i k_{3}}\right. \\
& \left.-\int_{0}^{t} y_{k}^{\prime}(s) \frac{e^{-i a(t-s)\left|k_{\perp}\right|^{2}-\sigma(t-s)+i k_{3}(-t+s)}}{i a\left|k_{\perp}\right|^{2}+\sigma+i k_{3}} d s\right] .
\end{aligned}
$$


We deduce from (4.5) with the Minkowski inequality that

$$
\begin{aligned}
\left\|\left(-i a \Delta_{\perp}+\partial_{z}\right) I\right\|_{2}^{2} \leqq & 4 \sum_{k}\left(\left|y_{k}(t)\right|^{2}+\left|y_{k}(0)\right|^{2} e^{-2 \sigma t}\right) \\
& +2 \sum_{k}\left(\int_{0}^{t}\left|y_{k}^{\prime}(s)\right| e^{-\sigma(t-s)} d s\right)^{2} \\
\leqq & 8 \rho_{0}^{2}+2\left(\int_{0}^{t}\left(\sum_{k}\left|y_{k}^{\prime}(s)\right|^{2} e^{-2 \sigma(t-s)}\right)^{\frac{1}{2}} d s\right)^{2} \\
\leqq & 8 \rho_{0}^{2}+2\left(\int_{0}^{t} e^{-\sigma(t-s)}\left(\sum_{k}\left|y_{k}^{\prime}(s)\right|^{2}\right)^{\frac{1}{2}} d s\right)^{2} \\
\leqq & 8 \rho_{0}^{2}+2 \frac{M_{1}^{2}}{\sigma^{2}} \equiv M_{2}^{2}
\end{aligned}
$$

where $M_{2}$ depend only on the coefficients of MB. Hence,

$$
\left\|\left(-i a \Delta_{\perp}+\partial_{z}\right)\left(X(x)-e^{i a t \Delta_{\perp}-\sigma t} X_{0}(x, y, z-t)\right)\right\|_{2} \leqq M_{2} .
$$

Let $\varphi \in C^{\infty}\left(T^{3}\right)$, then

$$
\begin{aligned}
\int_{T^{3}}\left(-i a \Delta_{\perp}+\partial_{z}\right) X(x) \cdot \varphi^{\star}(x) d x= & \int_{T^{3}}\left(-i a \Delta_{\perp}+\partial_{z}\right)\left(X(x)-e^{i a t \Delta_{\perp}-\sigma t} X_{0}\right) \varphi^{\star} \\
& +\int_{T^{3}}\left(-i a \Delta_{\perp}+\partial_{z}\right)\left(e^{i a t \Delta_{\perp}-\sigma t} X_{0}\right) \varphi^{\star} .
\end{aligned}
$$

Here all derivatives are in the weak sense. Now by (4.6) and (4.7):

$$
\begin{aligned}
\left|\int_{T^{3}}\left(-i a \Delta_{\perp}+\partial_{z}\right) X(x) \varphi^{\star}(x) d x\right| & \leqq M_{2}\|\varphi\|-\int_{T^{3}}\left(e^{i a t \Delta_{\perp}-\sigma t} X_{0}\right)\left(-i a \Delta_{\perp}+\partial_{z}\right) \varphi^{\star} d x \\
& \leqq M\|\varphi\|_{2}+e^{-\sigma t} \rho_{0}\left\|\left(-i a \Delta_{\perp}+\partial_{z}\right) \varphi\right\|_{2}
\end{aligned}
$$

Letting $t \rightarrow+\infty$ in (4.8) shows

$$
\left\|\left(-i a \Delta_{\perp}+\partial_{z}\right) X\right\|_{2} \leqq M
$$

or $\left(-i a \Delta_{\perp}+\partial_{z}\right) X \in L^{2}\left(T^{3}\right)$. We complete the proof.

Remark 4.1. Because of the multiplier $\left(\sigma+i k_{3}+i a\left|k_{\perp}\right|^{2}\right)^{-1}$, we do not have smoothing in all partial derivatives $\left(\left(k_{3}+a\left|k_{\perp}\right|^{2}\right)^{2}\right.$ does not bound $c|k|^{2}$ from above). We only have full smoothing in all derivatives if either $a=0$ which is the one-D in $z$ case or $k_{3}$ is absent which is the two-D in $(x, y)$ case.

Remark 4.2. Since $\left(-i a \Delta_{\perp}+\partial_{z}\right)$ is only a directional derivative, we are unable to show that $Y$ or $Z$ has smoothing in terms of $\left(-i a \Delta_{\perp}+\partial_{z}\right)$. In fact, applying $\left(-i a \Delta_{\perp}+\partial_{z}\right)$ formally to the $Y$ and $Z$ equation will generate $\nabla^{\perp}(X, Y, Z)$ terms, on which we have no control in $L^{2}$. 
Remark 4.3. In case of the two-D transverse (in $(x, y)) \mathrm{MB}$, we go to the $(Y, Z)$ equations to show $(Y, Z) \in H^{1}$ and iterate the above procedure to achieve $C^{\infty}$ smoothness of attractor $\mathscr{A}$ as in [6]. Moreover, the smooth attractor on the periodic domain has finite Hausdorff and fractal dimensions. The details of these calculations are carried out in [2].

Finally, we comment on some physical modifications on MB. The first is that due to imperfect mirror reflection and finite size cavity, the periodic boundary condition in the $z$ direction should be replaced by

$$
X(0, t)=R X(1, t-\Delta t),
$$

where $R \in(0,1)$ is the reflection coefficient of the mirrors, and $\Delta t>0$ is the delay time. However, one can make a linear change of variable in $z$ and $t$ (see [13]) so that the transformed $\mathrm{MB}$ is periodic $z$ and differs only in that the $\operatorname{Re}\left(X Y^{\star}\right)$ term in the $Z$ equation is replaced by $e^{a^{\prime} z} \operatorname{Re}\left(X Y^{\star}\right)$ for some constant $a^{\prime}>0$. The other is the inhomogeneous broadening effect of laser beams([13]), which amounts to replacing the $Y$ term in the $X$ equation by

$$
\int_{R^{1}} \varphi(\delta) Y(\delta, t, x, y, z) d \delta,
$$

and $(Y, Z)$ depending on the parameter $\delta$. If $\varphi$ is a delta function in $\delta$, we are back to (1.4)-(1.6). Incorporating both effects, we have the modified MB system:

$$
\begin{aligned}
X_{t}+X_{z}-i a \Delta_{\perp} X & =-\sigma X+\sigma \int_{R^{1}} \varphi(\delta) Y(\delta, t, x, y, z) d \delta, \\
Y_{t}(\delta, t, x, y, z) & =-(1+i \Omega) Y-Z X, \\
Z_{t}(\delta, t, x, y, z) & =-b Z+e^{a^{\prime} z} \operatorname{Re}\left(X Y^{\star}\right)-b r(x, y, z) .
\end{aligned}
$$

It is not hard to verify that if $\varphi \in L^{1}\left(R^{1}\right)$ or $L^{2}\left(R^{1}\right)$ our analysis on weak solutions carries over to system (4.9-4.11). We omit further details.

Acknowledgements The work of J.X. was partially supported by NSF grant DMS-9302830, and the Swedish Natural Science Research Council(NFR) grant F-GF 10448-301 at the Institut MittagLeffler during his visit there in the Fall of 1994 Both authors wish to thank Prof Bill Faris for helpful conversations and the Arizona Center for Mathematical Sciences (ACMS) for its stimulating environment and support ACMS is sponsored by AFOSR under contract F49620-94-1-0144DEF

\section{References}

1 Arechi, F T., Boccaletti, S, Ramazza, P L.: Phys Rev Lett. 70, 2277 (1993)

2 Birnir, B, Xin, J : The Global Attractor of the Transverse Maxwell-Bloch Equations 1995

3. Bourgain, J : Fourier Transform Restriction Phenomenon for Certain Lattice Subsets and Applications to Nonlinear Evolution Equations Part I, Schroedinger Equations J. Geometric and Functional Analysis 3, No 2, 107-156 (1993)

4 Caffarelli, L, Kohn, R., Nirenberg, L.: Partial Regularity of Suitable Weak Solutions of the Navier-Stokes Equations. Comm Pure Appl. Math, 35, 771-831 (1982)

5 Constantin, P, Foias, C.: Navier-Stokes Equations Chicago, IL: Univ of Chicago Press, 1988

6 Constantin, P, Foias, C, Gibbon, J.D : Finite Dimensional Attractor for the Laser Equations Nonlinearity 2, 241-269 (1989) 
7 Ginibre, J., Velo, G : On the global Cauchy problem for some nonlinear Schroedinger equations. Ann. Inst H Poincaré, 1, No. 4, 309-323 (1984)

8. Jacobsen, P K., Moloney, J.V, Newell, A.C., Indik, R.: Space-time dynamics of wide-gainsection lasers. Phys. Rev A 45, 8129 (1992)

9 Kato, $\mathrm{T}$ : On the nonlinear Schroedinger equations Ann Inst H Poincaré 46, No 1, 123-129 (1987)

10 Ladyzhenskaya, O A : The Mathematical Theory of Viscous Incompressible Flows 2nd ed, New York: Gordon and Breach, 1969

11. Lega, J, Jacobsen, P K., Moloney, J V , Newell, A C : Nonlinear Transverse Modes of Large Aspect Ratio Homogeneously Broadened Lasers II. Pattern Analysis Near and Beyond Threshold Phys Rev A, 49, No 5, 4201-4212 (1994)

12 Lega, J, Moloney, J.V , Newell, A C : Universal Description of Laser Dynamics Near Threshold Preprint, 1995

13 Lugiato, L A, Narducci, L.M., Bandy, D K., Tredicce, J R.: Single-mode approximation in laser physics: A critique and a proposed improvement Phys. Rev. A, 33, No. 2 (1986)

14 Newell, A.C, Moloney, J M.: Nonlinear Optics Reading, Mass.: Addison-Wesley, 1992

15 Newell, A C.: Patterns in Nonlinear Optics: A Paradigm Proceedings on NATO Advanced Research Workshop-Spatial/Temporal Patterns in Nonequilibrium Complex Systems, Santa Fe, New Mexico, 1993

16 Temam, R.: Infinite Dimensional Dynamical Systems in Mechanics and Physics Applied Math. Sci 68, Berlin Heidelberg, New York: Springer 1988

17 Tsutsumi, Y : $L^{2}$ solutions for nonlinear Schroedinger equations and nonlinear groups Funk Ekv. 30, 115-125 (1987)

18. Whitham, G B : Linear and Nonlinear Waves New York: Wiley, 1979

Communicated by A. Kupiainen 